Supporting Information

Palladium(II) Acetate in Pyridine as an Effective Catalyst for Highly Regioselective Hydroselenation of Alkynes

\author{
Ikuyo Kamiya, ${ }^{\dagger}$ Etsuyo Nishinaka,${ }^{\dagger}$ and Akiya Ogawa*, ${ }^{\ddagger}$ \\ Department of Chemistry, Faculty of Science, Nara Women's University, \\ Kitauoyanishi-machi, Nara 630-8506, Japan \\ Department of Applied Chemistry, Faculty of Engineering, Osaka Prefecture \\ University, Sakai, Osaka 599-8531, Japan \\ ogawa@chem.osakafu-u.ac.jp
}

\title{
List of Contents
}

General Comments

FIGURE 1 
General Comments. ${ }^{1} \mathrm{H}$ and ${ }^{13} \mathrm{C}$ NMR spectra $(300,400$ and $75 \mathrm{MHz}$, respectively) were determined by using $\mathrm{CDCl}_{3}$ as the solvent with $\mathrm{Me}_{4} \mathrm{Si}$ as the internal standard. Chemical shifts in ${ }^{1} \mathrm{H}$ and ${ }^{13} \mathrm{C}$ NMR were measured relative to $\mathrm{CDCl}_{3}$ and converted to $\delta$ $\left(\mathrm{Me}_{4} \mathrm{Si}\right)$ value by using $\delta\left(\mathrm{CDCl}_{3}\right)=7.26$ and $77.0 \mathrm{ppm}$, respectively. IR spectra were recorded on FT-IR instrument. Mass spectra were run by EI ionization $(70 \mathrm{eV})$.

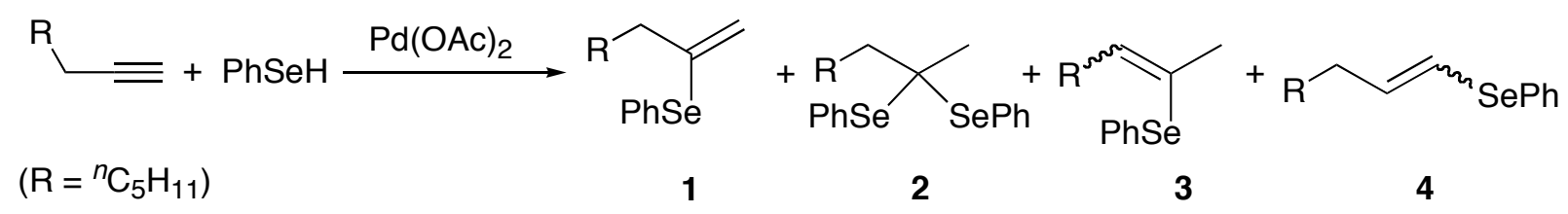

(a) $\stackrel{\Delta}{\Delta}$

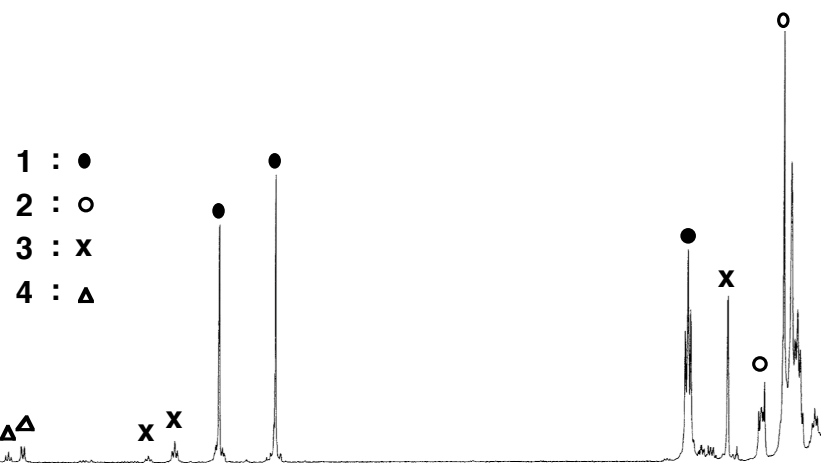

(b)

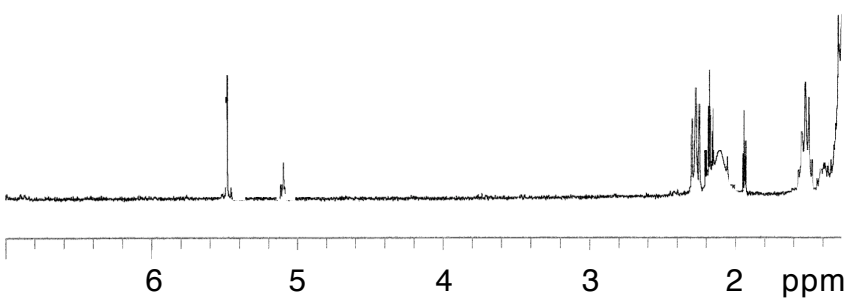

FIGURE 1. ${ }^{1} \mathrm{H}$ NMR Spectra of Crude Products from the $\mathrm{Pd}(\mathrm{OAc})_{2}$-Catalyzed Hydroselenation of 1-Octyne in THF (a) and in Pyridine (b) 
The following products are known compounds and CAS registry numbers are listed below.

2-Phenylseleno-1-octene (Table 2, entry 1) [63831-77-6].

2-Phenylseleno-3-phenyl-1-propene (Table 2, entry 4) [63831-80-1].

2-Phenylseleno-2-propen-1-ol (Table 2, entry 6) [104828-93-5].

4-Phenylseleno-4-hexenenitrile (Table 2, entry 2).

TLC: $\operatorname{Rf}=0.38($ hexane/ethyl acetate $=84 / 16)$.

Yellow oil; ${ }^{1} \mathrm{H}$ NMR (300 MHz, $\mathrm{CDCl}_{3}$ ) $\delta 1.89$ (quint, $J=7.2 \mathrm{~Hz}, 2 \mathrm{H}$ ), 2.33 (t, $J=7.1$ $\mathrm{Hz}, 2 \mathrm{H}), 2.43(\mathrm{t}, J=7.2 \mathrm{~Hz}, 2 \mathrm{H}), 5.25(\mathrm{~s}, 1 \mathrm{H}), 5.58(\mathrm{~s}, 1 \mathrm{H}), 7.31-7.56(\mathrm{~m}, 5 \mathrm{H}) ;{ }^{13} \mathrm{C}$ NMR $\left(75 \mathrm{MHz}, \mathrm{CDCl}_{3}\right) \delta 15.8,23.9,36.5,118.8,119.4,128.2,129.5,134.8,134.9,140.6 ; \mathrm{IR}$ ( $\mathrm{NaCl}) 3061,2936,2245,1611,1475,1437,1213,1022,912,877,741,692 \mathrm{~cm}^{-1}$. Anal. Calcd for $\mathrm{C}_{12} \mathrm{H}_{13} \mathrm{NSe}$ : C, 57.61; H, 5.24; N, 5.60. Found: C, 57.74; H, 5.21; N, 5.67.

2-Phenylseleno-1-hexene-1-ol (Table 2, entry 3).

TLC: $\operatorname{Rf}=0.41($ hexane/ethyl acetate $=64 / 36)$.

Yellow oil; ${ }^{1} \mathrm{H}$ NMR (300 MHz, $\left.\mathrm{CDCl}_{3}\right) \delta 1.44-1.64(\mathrm{~m}, 4 \mathrm{H}), 2.29(\mathrm{t}, J=7.1 \mathrm{~Hz}, 2 \mathrm{H})$, $3.31(\mathrm{~s}, 1 \mathrm{H}), 3.52(\mathrm{t}, J=6.3 \mathrm{~Hz}, 2 \mathrm{H}), 5.10(\mathrm{~s}, 1 \mathrm{H}), 5.51(\mathrm{~s}, 1 \mathrm{H}), 7.27-7.57(\mathrm{~m}, 5 \mathrm{H}) ;{ }^{13} \mathrm{C}$ NMR $\left(75 \mathrm{MHz}, \mathrm{CDCl}_{3}\right) \delta 26.0,32.7,39.0,62.7,117.1,129.1,130.1,130.5,136.0,144.8$; IR ( $\mathrm{NaCl}) 3308,3074,2936,2862,2370,1610,1578,1475,1437,1067,1022,870,739$, $691 \mathrm{~cm}^{-1}$. Anal. Calcd for $\mathrm{C}_{12} \mathrm{H}_{16} \mathrm{OSe}$ : C, 56.47; H, 6.32. Found: C, 56.18; H, 6.22. 
2-Phenylseleno-5-methyl-1-hexene (Table 2, entry 5).

TLC: $\mathrm{Rf}=0.28$ (hexane).

Yellow oil; ${ }^{1} \mathrm{H}$ NMR $\left(300 \mathrm{MHz}, \mathrm{CDCl}_{3}\right) \delta 0.77(\mathrm{~d}, J=6.3 \mathrm{~Hz}, 6 \mathrm{H}), 1.34(\mathrm{dt}, J=6.6,6.6$ $\mathrm{Hz}, 2 \mathrm{H}), 1.47$ (quint, $J=6.6 \mathrm{~Hz}, 1 \mathrm{H}), 2.21(\mathrm{t}, J=8.0 \mathrm{~Hz}, 2 \mathrm{H}), 5.03(\mathrm{~s}, 1 \mathrm{H}), 5.42(\mathrm{~s}, 1 \mathrm{H})$, 7.19-7.50 (m, 5H); ${ }^{13} \mathrm{C}$ NMR $\left(75 \mathrm{MHz} \mathrm{CDCl}_{3}\right) \delta 22.3,27.3,36.2,37.9,116.0,127.8$, 129.1, 129.3, 134.9, 144.0; IR (NaCl) 3067, 2955, 2928, 2868, 2852, 1609, 1580, 1475, 1468, 1366, 1022, 739, $691 \mathrm{~cm}^{-1}$. Anal. Calcd for $\mathrm{C}_{13} \mathrm{H}_{18} \mathrm{Se}: \mathrm{C}, 61.66 ; \mathrm{H}, 7.16$. Found: C, $61.93 ; \mathrm{H}, 7.28$.

1-Phenylseleno-1-(p-methoxyphenyl)-ethene (Table 2, entry 7).

This hydroselenation product is unstable and makes it difficult to isolate. Therefore, only ${ }^{1} \mathrm{H}$ NMR spectra data is shown, as a reference.

TLC: $\mathrm{Rf}=0.42$ (hexane/ethyl acetate $=80 / 20)$.

Yellow oil; ${ }^{1} \mathrm{H}$ NMR (400 MHz, $\left.\mathrm{CDCl}_{3}\right) \delta 3.83(\mathrm{~s}, 3 \mathrm{H}), 5.32$ (s, 1H), $5.84(\mathrm{~s}, 1 \mathrm{H}), 7.16-$ $7.53(\mathrm{~m}, 9 \mathrm{H})$. 